\title{
El patrimonio arqueológico en los libros de texto de Educación Secundaria*
}

\section{Archaeological heritage in Secondary Education textbooks}

\section{Antonio J. Meseguer Gil Laura Arias Ferrer Alejandro Egea Vivancos}

Universidad de Murcia

Resumen: En este trabajo se analiza cómo es introducido el patrimonio arqueológico en los libros de texto de $1 .^{\circ}$ de ESO y la función que este posee para poder analizar así el aprovechamiento cognitivo que se demanda sobre ellos. Se utilizan como muestra seis libros de texto de $1 .^{\circ} \mathrm{ESO}$, pertenecientes a tres editoriales distintas durante la vigencia de las normativas LOE y LOMCE. Se realiza una investigación con técnicas cualitativas para analizar el contenido de la muestra de manera contextualizada. Los resultados arrojan una creciente presencia del patrimonio arqueológico en los libros de texto y una mayor variedad de formatos, aunque las acciones demandadas sobre este siguen caracterizándose por su bajo nivel cognitivo.

Palabras clave: Educación patrimonial, Evaluación de libros de texto, Investigación en Ciencias Sociales, Indagación, Investigación Cualitativa.

Abstract: The aim of this paper is to analyze how archaeological heritage is introduced and used in Secondary Education History textbooks (Year 7, 10-11 year old students). To get that aim, it has been selected a sample of six textbooks edited by three different publishers under different education laws (LOE and LOMCE). A qualitative research was conducted in order to analyze the sample in a contextualized way. Results show an increasing presence of archaeological heritage in textbooks and in a wider range of format, although the actions demanded regarding the archaeological heritage is still characterized by its low cognitive character, that requires very little reasoning by the students.

Keywords: Heritage education, Textbook evaluation, Social Science research, Inquiry, Qualitative research.

(Fecha de recepción: noviembre, 2017, y de aceptación: diciembre, 2017)

DOI: 10.7203/DCES.33.11003

* Investigación desarrollada en el marco del proyecto "La evaluación de las competencias y el desarrollo de capacidades cognitivas sobre Historia en Educación Secundaria Obligatoria" (EDU2015-65621-C3-2-R), financiado por el Ministerio de Economía y Competitividad de España y Fondos FEDER. 


\section{Introducción}

A pesar de su carácter interdisciplinar, la arqueología suele llegar a las aulas de la mano de la materia de Historia. Su metodología facilita que sea el estudiante el que construya su propio conocimiento y desarrolle las denominadas habilidades del pensamiento histórico ya definidas por Seixas y Morton (2013) (Egea y Arias, 2017; Wearing, Case y Edwards, 2011). Esto se debe al alto carácter procedimental que posee la disciplina arqueológica, junto al que cabría destacar otros beneficios como son la interdisciplinareidad de su método, las dinámicas de trabajo en equipo que genera o el vínculo con el patrimonio cultural que establece, entre otros (Corbishley, 2011; Egea, Arias y Pernas, 2017; Henson, 2004; Hernández, 2003; Santacana, 1997, 2015). Además, no hay que olvidar que existe una predisposición positiva hacia la arqueología por parte de los estudiantes, derivada de la concepción romántica que posee, que actúa como catalizador para un acercamiento provechoso al método y las actitudes que promueve (Feliu y Hernàndez, 2011). Y es que esta disciplina posee lo que Henson denominó como "wow factor" (2009, p. 7).

En España, las experiencias educativas que centran su atención en la introducción de la arqueología en el aula crecen en número (Bardavio, Gatell y González, 2004; Egea y Arias, 2013; Pallarés, Carrión y Sánchez, 2017; Santacana, 1997). Pero, sin embargo todavía se pueden considerar casos aislados en el proceso de enseñanza-aprendizaje. Se podría aducir a los costes que el desa- rrollo de una excavación arqueológica simulada en los propios centros escolares puede ocasionar o a las múltiples gestiones necesarias para posibilitar la participación del alumnado en excavaciones ordinarias.

Dentro de esta misma consideración, por los nexos que guarda con la metodología arqueológica, se podría encuadrar la denominada "didáctica del objeto" (Durbin, Morris y Wilkinson, 1990; Santacana y Llonch, 2012). Ésta permite introducir la metodología arqueológica en el aula de una manera motivadora y evitando los costes (económicos, materiales y temporales) que una excavación arqueológica puede conllevar. El objeto pasa a ser el vehículo de análisis y reflejo de la sociedad y el contexto en el que surge (Egea, Pernas y Arias, 2014). En este caso se está ante la dificultad de que los centros escolares (y se podría añadir museos y centros de interpretación como nexo entre escuela y patrimonio arqueológico) posean colecciones de objetos y réplicas que permitan la interacción de los estudiantes con el patrimonio arqueológico.

Sin embargo, para introducir la arqueología en el aula no es necesario introducir objetos arqueológicos de manera física o realizar excavaciones arqueológicas -reales o simuladas-, pues los propios materiales empleados de manera habitual en el ámbito escolar pueden utilizarse siguiendo una metodología de indagación asociada a la propia labor del arqueólogo. Los propios libros de texto pueden ser la base sobre la que realizar indagaciones de corte arqueológico si estos dejan de ser considerados como protagonistas indiscutibles 
del proceso de enseñanza-aprendizaje y pasan a ser utilizados como instrumento de apoyo (Prats, 2012), contenedor de diversos recursos que pueda facilitar el acceso a la información de los estudiantes. Bajo estas premisas, los libros de texto se convertirían en elementos a analizar y no en documentos a leer, subrayar y memorizar.

De ahí las principales cuestiones que vertebran esta investigación: ¿Con qué frecuencia está presente el patrimonio arqueológico en los libros de texto? ¿Qué estrategias o actividades aparecen asociadas?

Se insiste en centrar la atención en el caso concreto de los libros de texto ya que estos se muestran, por el momento, como el recurso más presente en las aulas españolas (Burguera, 2006; Cuenca, 2002; Martínez, 2011; Prats, 2012). Una observación detallada de los mismos permitirá realizar un primer análisis y valoración acerca de cómo es introducido el patrimonio arqueológico en las aulas de Educación Secundaria, lo que facilitará el posterior desarrollo de propuestas de mejora que puedan dar solución a las carencias/problemas detectados.

\section{Marco Teórico}

\section{El patrimonio arqueológico}

Para poder responder a las preguntas de investigación propuestas es necesario definir en primer lugar qué se entiende por patrimonio arqueológico. En este sentido, este análisis parte de la concepción disciplinar del patrimonio arqueológico derivada del artículo 40.1 de la Ley del Patrimonio
Histórico Español. Este designa como tal a "aquellos bienes muebles o inmuebles susceptibles de ser estudiados con metodología arqueológica, hayan sido o no extraídos y tanto si se encuentran en la superficie o en el subsuelo, en el mar territorial o en la plataforma continental" (Ley 16/1985, p. 20347). Dicha definición es compartida en términos generales por la UNESCO en el Charter for the Protection and Management of Archaeological Heritage, aprobado por el International Council on Monument and Sites (ICOMOS) en 1990. En su artículo 1 se identifica el patrimonio arqueológico con "all vestiges of human existence" junto a "all manifestations of human activity, abandoned structures and remains of all kinds (...) together with all the portable cultural material associated with them". Dicha definición fue posteriormente precisada en el Convenio Europeo sobre la Protección del Patrimonio Arqueológico realizado en La Valetta el 16 de enero de 1992 y revisado y ratificado por el Gobierno de España el 20 de julio de 2011. Dicho convenio señala en su artículo 1.2. que "se consideran elementos del patrimonio arqueológico todos los vestigios, objetos y cualesquiera otras trazas de manifestaciones humanas de épocas pasadas (i.) cuya preservación y estudio permitan reconstruir la historia de la humanidad y su relación con el medio ambiente natural; (ii.) respecto de los cuales los principales medios de información sean las excavaciones o descubrimientos, así como otros métodos de investigación relativos a la humanidad y a su entorno". Junto a esto, en su artículo 1.3. dicho convenio precisa que este 
"incluye las estructuras, construcciones, grupos arquitectónicos, lugares de asentamiento, objetos muebles, monumentos de otra naturaleza, así como su contexto, localizados en tierra o bajo el agua".

Sin embargo, no hay que olvidar que el empleo de métodos y fuentes asociadas a la arqueología está estrechamente ligado con el propio proceso de construcción del conocimiento histórico y que no constituye un fin en sí mismo (Bardavio y González, 2003). Cabría igualmente añadir el valor estético y artístico que gran parte de este patrimonio arqueológico posee, pudiendo observar la estrecha interrelación que la disciplina arqueológica posee con la historia y la historia del arte (entre otras). La clasificación de un elemento patrimonial en relación a cada una de estas disciplinas se verá más determinado por el enfoque y metodología de análisis a aplicar que por las características propias del elemento en sí mismo (Cuenca, 2002).

Es por ello que en este análisis específico, se opta por incluir como patrimonio arqueológico aquellos bienes arquitectónicos, escultóricos y pictóricos realizados por las sociedades antiguas, así como los objetos, entendiendo como tal cualquier resto material tangible propio de estas sociedades (Feliu y Hernàndez, 2011).

\section{Los libros de texto como recurso educativo}

Como ya se ha señalado anteriormente, la selección del recurso específico sobre el que centrar esta investigación (el libro de texto) se basa en el hecho de que este es el material curricular más extendido, estudiado y aceptado (Cuenca, 2002), consolidado como el intermediario por excelencia entre el currículo y la legislación de los estados y su concreción en el aula (Raja y Miralles, 2015). Según las conclusiones de Schug, Western y Enochs (1997), recogidas y analizadas por Cuenca y Estepa (2003), habría cuatro variables que pueden ayudar a entender esta hegemonía del libro de texto: la relación entre costes y beneficios para el docente en cuanto al tiempo empleado en la preparación de sus clases, el interés económico editorial, la gran oferta y actualización continuada que existe $y$, por último, la propia demanda social.

Es por ello que el análisis de los libros de texto se constituye como fundamental en la investigación educativa ya que viene a reflejar gran parte de los contenidos y procedimientos que se desarrollan dentro del aula. En España, dicha preocupación sobre la dimensión didáctica del libro de texto surge a finales de los años 90, especialmente encabezada por los trabajos de Valls (1998, 1999, 2001, 2008). Muchos otros trabajos han seguido la estela de estas primeras aproximaciones al análisis de contenido y valoración del uso de este recurso, muchos de ellos recopilados por Gómez, Cózar y Miralles (2014), y a los que se suman los recientes análisis sobre la presencia en los libros de texto de diversos aspectos específicos como son el pensamiento histórico (Sáiz y Colomer, 2014), las competencias clave (Sáiz, 2014) o el tratamiento de periodos específicos de la historia (Rausell, 
2017; Sánchez, Arias y Egea, 2016) o la función de elementos específicos que los conforman, como son las imágenes (Bel, 2017; Gámez, 2016).

Estos estudios en general advierten de la excesiva carga conceptual que estos poseen asociada además a acciones centradas en la memorización y repetición (Bel, 2017; Sáiz, 2014; Sáiz y Colomer, 2014). En los casos en los que el docente se aferra al contenido de estos (Ferreras y Jiménez, 2013), no aporta al alumnado las herramientas para llevar a cabo una enseñanza crítica y reflexiva. Además, la velocidad de los cambios actuales deja desfasados gran parte de los enfoques y contenidos recogidos en los manuales (Raja y Miralles, 2015). Este hecho, didácticamente hablando, sume a la práctica docente en una espiral que refuerza su limitación conceptual (Valls, 2008), ratificadas cuando el uso del libro de texto no se flexibiliza ni complementa.

El tradicional peso que lo conceptual posee en los libros de texto hace que la asociación de acciones de vertiente más procedimental ligadas a la introducción del patrimonio arqueológico se intuya escasa. Diversos estudios que analizan la presencia del patrimonio cultural en los libros de texto señalan cómo este suele aparecer representado como material ilustrativo del contenido sobre el que apenas se exige demanda procedimental o sobre el que prima una valoración estética y artística de corte academicista (Cuenca, 2002; Cuenca y Estepa, 2003; Cuenca, Estepa y Martín, 2017; Cuenca y López, 2014; Estepa, Ferreras, López y Morón, 2015; Hernàndez, 2003; Hernández y Guillén,
2017; López, 2014; López y Cuenca, 2011; Trabajo y Cuenca, 2014; Sánchez y Martínez, 2015).

Estos estudios muestran la escasa interacción que normalmente se realiza sobre los recursos en general, y el patrimonio en particular, que aparecen recogidos en el libro de texto. Ya se ha precisado previamente el enorme potencial que el patrimonio de carácter arqueológico posee como fuente de conocimiento si este se asocia con estrategias asociadas al propio método del arqueólogo. Un análisis específico sobre el caso del patrimonio arqueológico permitirá realizar un diagnóstico inicial que fundamente futuras intervenciones al respecto, con el objetivo último de promover la mejora del proceso de enseñanza-aprendizaje.

\section{Marco empírico}

Este trabajo de investigación posee un corte eminentemente cualitativo, basado en las técnicas de análisis de contenido (Andreu, 2002), y pretende dar respuesta a dos objetivos específicos asociados a las preguntas de investigación previamente planteadas:

1) Identificar cómo aparece representado el patrimonio arqueológico en los libros de texto.

2) Analizar qué acciones cognitivas se demandan en relación al patrimonio arqueológico.

\section{Contexto y muestra}

El estudio se centra en el análisis de seis libros de texto publicados durante los periodos de vigencia de dos legisla- 
ciones educativas distintas y consecutivas: LOE (Ley Orgánica 2/2006; Decreto 291/2007) y LOMCE (Ley Orgánica 8/2013; Decreto 220/2015). De cada una de las tres editoriales seleccionadas se analizaron dos manuales, cada uno de ellos correspondiente al periodo de vigencia de cada ley. El universo del que se seleccionó la muestra es el de las editoriales con mayor difusión dentro de la Región de Murcia, entre las cuales se encuentran Oxford (Carrasco et al. 2007; Navarro y Alcolea, 2015), Santillana (Adiego et al. 2015; Grence et al. 2011) y Vicens Vives (Albet, Benejam, García y Gatell, 2007; Albet et al. 2015).

El estudio se centró únicamente en 1. ${ }^{\circ}$ de la ESO, ya que el patrimonio histórico-artístico y arqueológico aparece tradicionalmente vinculado a los bloques de Prehistoria e Historia Antigua, disminuyendo con el avance cronológico de los contenidos (Cuenca y Estepa, 2003; Meseguer, Caballero, Arias y Egea, 2017).

\section{Instrumento de recogida de datos y procedimientos de análisis}

Se diseñó ad hoc un instrumento para la recogida y análisis de datos, que fueron almacenados en una hoja de cálculo (Microsoft Excel). En este análisis, las unidades de muestreo estaban compuestas por todas las apariciones del patrimonio arqueológico en los libros de texto, en cualquiera de los formatos posibles. El número de registros obtenidos a este respecto fue de $N=1246$. Cómo este patrimonio era presentado y la acción asociada al mismo constituye- ron las unidades de registro del instrumento diseñado.

Estas unidades de registro se referían al formato en el que el patrimonio era representado, diferenciando entre si este era presentado sin modificación alguna (y por lo tanto podía constituir una fuente primaria) o si era el resultado de una interpretación o descripción que trascendía a la mera exposición de la fuente primaria original. Se considera pues fuente primaria a los elementos coetáneos al periodo al que ilustran y que muestran el elemento patrimonial seleccionado sin alteración alguna. Estos aparecen en todos los casos analizados en formato imagen. En este caso, se diferencia entre "objeto", "pintura", "escultura" y "arquitectura". Por el contrario, como fuente secundaria se incluye "recreación" (todo aquel elemento patrimonial que ha sido reconstruido, interpretado o utilizado como soporte para añadir información o contenidos adicionales como, por ejemplo, vocabulario específico), "mapa" (cuando este forma parte de representaciones cartográficas con la intencionalidad de mostrar su distribución o localización) y "texto" (cuando este ha sido mencionado y descrito por otros autores -ya sea en textos clásicos, historiográficos y/o literarios-). Por último, dentro de la categoría de fuentes secundarias se ha añadido la categoría de "fotografía", referida a aquellas ocasiones en las que el objeto principal es la composición en sí y no el patrimonio arqueológico. Junto a esto, se describía el lugar dónde este elemento patrimonial había sido incorporado, pudiendo encontrarse en el texto principal, en hipertexto o 
en una actividad. El análisis de estos aspectos permitirían dar respuesta al objetivo 1 de esta investigación.

A continuación, se llevó a cabo su clasificación según la función que cada elemento poseía, diferenciando entre si este ilustraba el texto ("ilustra") o por el contrario era utilizado como recurso sobre el que llevar a cabo alguna acción ("demanda acción"). Si se correspondía con este segundo caso, se procedía a analizar su aprovechamiento cognitivo. Para ello se clasificaron las diferentes acciones demandadas en base a las pautas establecidas por Sáiz (2014) en anteriores análisis, diferenciándose entre un aprovechamiento cognitivo "bajo" (localizar y reproducir información proporcionada por el libro de texto), "medio" (explicar la información obtenida y compararla con otras fuentes) y "alto" (evaluar y crear nueva información). Dichos niveles de aprovechamiento se pueden equiparar a aquellos establecidos por Bloom (1956) y que diferencian entre acciones de menor y mayor demanda cognitiva (Anderson y Krathwohl, 2001), avanzando desde recordar, entender, aplicar, analizar, evaluar y crear. Esta clasificación fue posible gracias a la previa codificación de las acciones demandadas en cada uno de los enunciados. Así, se crearon las subcategorías de "Identificar"/"Describir" (bajo),"Comparar"/“Deducir"/"Explicar" (medio) e "Investigar"/“Analizar" (alto). Un elemento patrimonial sobre el que se demandara acción podía ir vinculado a más de una acción. El análisis de estas categorías permitiría dar respuesta al objetivo 2 de la investigación.

\section{Resultados}

La representación del patrimonio arqueológico en los libros de texto

Para dar respuesta al objetivo 1 de esta investigación se analiza en primer lugar cómo el patrimonio arqueológico es representado en los libros de texto. En este sentido, el Cuadro I muestra el total de registros asociados al patrimonio arqueológico que aparecen en los libros de texto de $1 .^{\circ}$ de ESO analizados.

\section{Cuadro I \\ Distribución de registros por tipos de fuentes}

Fuente: Elaboración propia

\begin{tabular}{|c|c|c|c|c|c|c|c|c|c|c|c|c|}
\hline \multirow{3}{*}{ Categorías } & \multicolumn{6}{|c|}{ LOE } & \multicolumn{6}{|c|}{ LOMCE } \\
\hline & \multicolumn{2}{|c|}{ Oxford } & \multicolumn{2}{|c|}{ Santillana } & \multicolumn{2}{|c|}{ V. Vives } & \multicolumn{2}{|c|}{ Oxford } & \multicolumn{2}{|c|}{ Santillana } & \multicolumn{2}{|c|}{ V. Vives } \\
\hline & $n$ & $\%$ & $n$ & $\%$ & $n$ & $\%$ & $n$ & $\%$ & $n$ & $\%$ & $n$ & $\%$ \\
\hline F. Primaria & 98 & 72.6 & 167 & 73.6 & 185 & 78.7 & 133 & 74.3 & 125 & 72.3 & 219 & 73.7 \\
\hline F. Secundaria & 37 & 27.4 & 60 & 26.4 & 50 & 21.3 & 46 & 25.7 & 48 & 27.7 & 78 & 26.3 \\
\hline Total & \multicolumn{2}{|c|}{135} & \multicolumn{2}{|c|}{227} & \multicolumn{2}{|c|}{235} & \multicolumn{2}{|c|}{179} & \multicolumn{2}{|c|}{173} & \multicolumn{2}{|c|}{297} \\
\hline
\end{tabular}


Se comprueba cómo en general el patrimonio arqueológico aparece más usualmente representado como fuente primaria. Aunque su presencia aumenta en los libros de texto de la LOMCE de las editoriales Oxford y Vicens Vives, los porcentajes de representación en uno $\mathrm{u}$ otro formato no se ven notablemen- te modificados. Pese a que la editorial Santillana haya reducido el número de elementos patrimoniales a incorporar, este sigue siendo superior al que la editorial Oxford presentaba en su edición anterior.

En el caso de qué patrimonio es representado cuando este es introdu-

\section{Cuadro II \\ Distribución de registros por fuentes primarias atendiendo al soporte utilizado}

(Fuente: Elaboración propia)

\begin{tabular}{|c|c|c|c|c|c|c|c|c|c|c|c|c|}
\hline \multirow{3}{*}{ Categorías } & \multicolumn{6}{|c|}{ LOE } & \multicolumn{6}{|c|}{ LOMCE } \\
\hline & \multicolumn{2}{|c|}{ Oxford } & \multicolumn{2}{|c|}{ Santillana } & \multicolumn{2}{|c|}{ V. Vives } & \multicolumn{2}{|c|}{ Oxford } & \multicolumn{2}{|c|}{ Santillana } & \multicolumn{2}{|c|}{ V. Vives } \\
\hline & $n$ & $\%$ & $n$ & $\%$ & $n$ & $\%$ & $n$ & $\%$ & $n$ & $\%$ & $n$ & $\%$ \\
\hline Arquitectura & 22 & 22.4 & 58 & 34.7 & 41 & 22.2 & 41 & 30.8 & 37 & 29.6 & 42 & 19.2 \\
\hline Escultura & 32 & 32.7 & 36 & 21.6 & 49 & 26.5 & 32 & 24.1 & 30 & 24.0 & 67 & 30.6 \\
\hline Pintura & 23 & 23.5 & 16 & 9.6 & 37 & 20 & 30 & 22.6 & 19 & 15.2 & 50 & 22.8 \\
\hline Objeto & 21 & 21.4 & 57 & 34.1 & 58 & 31.4 & 30 & 22.6 & 39 & 31.2 & 60 & 27.4 \\
\hline Total & \multicolumn{2}{|c|}{98} & \multicolumn{2}{|c|}{167} & \multicolumn{2}{|c|}{185} & \multicolumn{2}{|c|}{133} & \multicolumn{2}{|c|}{125} & \multicolumn{2}{|c|}{219} \\
\hline
\end{tabular}

\section{Cuadro III}

\section{Distribución de registros por fuentes secundarias}

(Fuente: Elaboración propia)

\begin{tabular}{|c|c|c|c|c|c|c|c|c|c|c|c|c|}
\hline \multirow{3}{*}{ Categorías } & \multicolumn{6}{|c|}{ LOE } & \multicolumn{6}{|c|}{ LOMCE } \\
\hline & \multicolumn{2}{|c|}{ Oxford } & \multicolumn{2}{|c|}{ Santillana } & \multicolumn{2}{|c|}{ V. Vives } & \multicolumn{2}{|c|}{ Oxford } & \multicolumn{2}{|c|}{ Santillana } & \multicolumn{2}{|c|}{ V. Vives } \\
\hline & $n$ & $\%$ & $n$ & $\%$ & $n$ & $\%$ & $n$ & $\%$ & $n$ & $\%$ & $n$ & $\%$ \\
\hline Recreación & 26 & 70.3 & 44 & 73.3 & 43 & 86 & 29 & 63 & 25 & 52.1 & 62 & 79.5 \\
\hline Mapa & 7 & 18.9 & 9 & 15 & 6 & 12 & 6 & 13 & 5 & 10.4 & 9 & 11.5 \\
\hline Texto & 4 & 10.8 & 3 & 5 & 1 & 2 & 8 & 17.5 & 8 & 16.7 & 7 & 9 \\
\hline Fotografía & 0 & 0 & 4 & 6.7 & 0 & 0 & 3 & 6.5 & 10 & 20.8 & 0 & 0 \\
\hline Total & \multicolumn{2}{|c|}{37} & \multicolumn{2}{|c|}{60} & \multicolumn{2}{|c|}{50} & \multicolumn{2}{|c|}{46} & \multicolumn{2}{|c|}{48} & \multicolumn{2}{|c|}{78} \\
\hline
\end{tabular}




\section{Cuadro IV \\ Distribución de registros según la ubicación \\ del elemento analizado en el discurso}

(Fuente: Elaboración propia)

\begin{tabular}{|c|c|c|c|c|c|c|c|c|c|c|c|c|}
\hline \multirow{3}{*}{ Categorías } & \multicolumn{6}{|c|}{ LOE } & \multicolumn{6}{|c|}{ LOMCE } \\
\hline & \multicolumn{2}{|c|}{ Oxford } & \multicolumn{2}{|c|}{ Santillana } & \multicolumn{2}{|c|}{ V. Vives } & \multicolumn{2}{|c|}{ Oxford } & \multicolumn{2}{|c|}{ Santillana } & \multicolumn{2}{|c|}{ V. Vives } \\
\hline & $n$ & $\%$ & $n$ & $\%$ & $n$ & $\%$ & $n$ & $\%$ & $n$ & $\%$ & $n$ & $\%$ \\
\hline Texto & 64 & 47.4 & 79 & 34.8 & 106 & 45.1 & 54 & 30.2 & 55 & 31.8 & 115 & 38.7 \\
\hline Hipertexto & 53 & 39.3 & 116 & 51.1 & 94 & 40.0 & 94 & 52.5 & 93 & 53.8 & 155 & 52.2 \\
\hline Actividad & 18 & 13.3 & 32 & 14.1 & 35 & 14.9 & 31 & 17.3 & 25 & 14.5 & 27 & 9.1 \\
\hline Total & \multicolumn{2}{|c|}{135} & \multicolumn{2}{|c|}{227} & \multicolumn{2}{|c|}{235} & \multicolumn{2}{|c|}{179} & \multicolumn{2}{|c|}{173} & \multicolumn{2}{|c|}{297} \\
\hline
\end{tabular}

cido como fuente primaria (Cuadro II), en general se observa cierta igualdad en cuanto al soporte escogido, con porcentajes similares entre "Arquitectura", "Escultura", "Pintura" y "Objeto".

En el caso de la forma de representación utilizada cuando se trata de una fuente secundaria (Cuadro III), es mayoritario el uso de recreaciones, aunque la frecuencia de las mismas se haya visto reducida en todos los casos en los ejemplares de la LOMCE. La inclusión del patrimonio en representaciones cartográficas también pierde frecuencia, en beneficio de la incorporación de fuentes documentales (clásicas, historiográficas o literarias) que mencionen elementos del patrimonio arqueológico, o fotos generales de conjunto donde el elemento patrimonial queda relegado a un segundo plano.

Si se atiende a su ubicación en el discurso (Cuadro IV), las fuentes patrimoniales suelen ser incluidas con relación al texto e hipertexto, y es menos frecuente su aparición junto a activi- dades. Esta reducida presencia como acompañamiento a las actividades se mantiene a lo largo del tiempo. No así la vinculación de la imagen al texto, ya que parece observarse un aumento de la presencia de este tipo de recursos junto al hipertexto.

Acciones cognitivas asociadas al patrimonio arqueológico

En relación a la función que los elementos arqueológicos poseen en el texto, destaca su rol como "ilustrador" de contenidos por encima de aquellos casos en los que se demanda acción (Cuadro V), especialmente en los ejemplares de Oxford y Vicens Vives, siendo Santillana la editorial que más frecuentemente introduce el elemento patrimonial como recurso sobre el que demandar algún tipo de acción. Este rol de ilustrador de contenidos se refuerza en las ediciones LOMCE de Santillana y Vicens Vives, donde se observa un ligero descenso en 
la demanda de acciones pese a haber aumentado el número de elementos patrimoniales presentes.

Si se centra la atención exclusivamente en aquellos registros que demandan acción $(n=498)$, es posible analizar el aprovechamiento cognitivo que sugieren esas acciones demandadas (Cuadro VI). Un elemento patrimonial sobre el que se demanda acción puede ir asociado a más de una acción, es por ello que se cuenta con un total de $n=981$ registros asociados a acciones. De ellas, un $50.8 \%(\mathrm{n}=498)$ son caracterizadas por su nivel de aprovechamiento bajo, un $42.8 \%(\mathrm{n}=420)$ por su nivel medio y un $6.4 \%(\mathrm{n}=63)$ por su nivel alto. Se observa cierta diferenciación atendiendo al momento de publicación del ejemplar. Así, entre libros de texto LOE se detecta un $54.9 \%(n=268)$ de acciones de nivel bajo, un $41.6 \%(\mathrm{n}=203)$ de nivel medio, y un $3.5 \%(\mathrm{n}=17)$ de nivel alto. Por el contrario, los ejemplares LOMCE presentan una reducción de acciones de bajo aprovechamiento cognitivo, aunque siguen siendo las más numerosas
( $46.7 \%, \mathrm{n}=230$ ), y aumentan las de nivel medio ( $44 \% \mathrm{n}=217) \mathrm{y}$, especialmente, las de un nivel alto $(9.4 \%, \mathrm{n}=46)$. Este incremento se produce especialmente en aquellas actividades que utilizan el elemento patrimonial como elemento de motivación para "investigar", que pasan de constituir un $1 \%$ de las acciones $(\mathrm{n}=5)$ a representar el $6.3 \%(\mathrm{n}=31)$.

Si se atiende a su evolución por editoriales, en general se observa que la editorial Oxford introduce menos acciones de bajo aprovechamiento cognitivo que las restantes editoriales, tanto en su ejemplar LOE como LOMCE, aunque estas siguen siendo abundantes. De la misma manera, esta editorial es también la que más acciones asociadas a un aprovechamiento cognitivo alto incorpora.

\section{Discusión de los resultados}

A raíz de los resultados obtenidos, parece detectarse una notable presencia del patrimonio arqueológico en los libros de texto. Esta importancia

\section{Cuadro V}

\section{Distribución de registros atendiendo a la función en el texto}

(Fuente: Elaboración propia)

\begin{tabular}{|c|c|c|c|c|c|c|c|c|c|c|c|c|}
\hline \multirow{3}{*}{ Categorías } & \multicolumn{6}{|c|}{ LOE } & \multicolumn{6}{|c|}{ LOMCE } \\
\hline & \multicolumn{2}{|c|}{ Oxford } & \multicolumn{2}{|c|}{ Santillana } & \multicolumn{2}{|c|}{ V. Vives } & \multicolumn{2}{|c|}{ Oxford } & \multicolumn{2}{|c|}{ Santillana } & \multicolumn{2}{|c|}{ V. Vives } \\
\hline & $n$ & $\%$ & $n$ & $\%$ & $n$ & $\%$ & $n$ & $\%$ & $n$ & $\%$ & $n$ & $\%$ \\
\hline Ilustra & 92 & 68.2 & 115 & 50.7 & 128 & 55.2 & 119 & 66.5 & 94 & 54.3 & 196 & 66.2 \\
\hline Demanda acción & 43 & 31.8 & 112 & 49.3 & 104 & 44.8 & 60 & 33.5 & 79 & 45.7 & 100 & 33.8 \\
\hline Total & \multicolumn{2}{|c|}{135} & \multicolumn{2}{|c|}{227} & \multicolumn{2}{|c|}{232} & \multicolumn{2}{|c|}{179} & \multicolumn{2}{|c|}{173} & \multicolumn{2}{|c|}{296} \\
\hline
\end{tabular}




\section{Cuadro VI \\ Distribución de registros por acciones demandadas y aprovechamiento cognitivo}

(Fuente: Elaboración propia)

\begin{tabular}{|c|c|c|c|c|c|c|c|c|c|c|c|c|c|}
\hline \multirow{3}{*}{\multicolumn{2}{|c|}{$\begin{array}{l}\text { Categorías } \\
\text { Aprovechamiento } \\
\text { cognitivo / Acción }\end{array}$}} & \multicolumn{6}{|c|}{ LOE } & \multicolumn{6}{|c|}{ LOMCE } \\
\hline & & \multicolumn{2}{|c|}{ Oxford } & \multicolumn{2}{|c|}{ Santillana } & \multicolumn{2}{|c|}{ V. Vives } & \multicolumn{2}{|c|}{ Oxford } & \multicolumn{2}{|c|}{ Santillana } & \multicolumn{2}{|c|}{ V. Vives } \\
\hline & & $n$ & $\%$ & $n$ & $\%$ & $n$ & $\%$ & $n$ & $\%$ & $n$ & $\%$ & $n$ & $\%$ \\
\hline \multirow{2}{*}{ Bajo } & Identificar & 23 & 33.8 & 62 & 32.3 & 53 & 23.2 & 13 & 11.7 & 43 & 26.7 & 54 & 24.4 \\
\hline & Describir & 12 & 17.6 & 45 & 23.4 & 73 & 32 & 26 & 23.4 & 31 & 19.3 & 63 & 28.5 \\
\hline \multirow{3}{*}{ Medio } & Comparar & 12 & 17.6 & 9 & 4.7 & 27 & 11.8 & 13 & 11.7 & 7 & 4.3 & 11 & 5 \\
\hline & Deducir & 4 & 5.9 & 35 & 18.2 & 48 & 21.1 & 26 & 23.4 & 29 & 18 & 51 & 23.1 \\
\hline & Explorar & 11 & 16.2 & 35 & 18.2 & 22 & 9.6 & 19 & 17.1 & 35 & 21.7 & 26 & 11.8 \\
\hline \multirow[t]{2}{*}{ Alto } & Analizar & 5 & 7.4 & 4 & 2.1 & 3 & 1.3 & 1 & 0.9 & 3 & 1.9 & 11 & 5 \\
\hline & Investigar & 1 & 1.5 & 2 & 1 & 2 & 0.9 & 13 & 11.7 & 13 & 8.1 & 5 & 2.3 \\
\hline Total & & \multicolumn{2}{|c|}{68} & \multicolumn{2}{|c|}{192} & \multicolumn{2}{|c|}{228} & \multicolumn{2}{|c|}{111} & \multicolumn{2}{|c|}{161} & \multicolumn{2}{|c|}{221} \\
\hline
\end{tabular}

se mantiene y refuerza en los últimos manuales publicados adscritos a la LOMCE, asociada al ligero incremento en la presencia de imágenes que progresivamente se viene detectando en los libros de texto españoles (Bel, 2017).

Mayoritariamente estos elementos son presentados en formato imagen a modo de fuentes primarias, es decir, sin modificación ni interpretación en su representación, y sin ser utilizadas como soporte para la introducción de información adicional, constituyendo entre el $72.6 \%-78.5 \%$ (LOE) y el $72.3 \%$ $74.3 \%$ (LOMCE). En estos casos, los elementos patrimoniales representados se ciñen a elementos arquitectónicos, escultóricos o pictóricos, todos ellos representados en similar proporción. Es de destacar cómo la cultura material (objetos) posee similar presencia (entre el 21.4\%-34.1\%) (Cuadro III).

En estos casos, el patrimonio suele aparecer de manera descontextualizada, por lo que su potencial en el proceso de construcción del conocimiento histórico se ve altamente reducido (López, 2014). Este aspecto se debe a la mayor incidencia del patrón de análisis estético que se produce sobre estas piezas, obviando en muchas ocasiones el componente arqueológico, que hace que se derive la observación de estos elementos hacia una acción descriptiva y no hacia un uso activo del patrimonio y en su valor como fuente de conocimiento. Se descuida así su capacidad para el acceso al conocimiento histórico (Rüsen, 1997). 
Cuando el elemento patrimonial es representado como fuente secundaria, este lo hace mayoritariamente a modo de reconstrucción o recreación, donde se incorporan elementos adicionales. Sin embargo, es de destacar cómo en las últimas ediciones analizadas asociadas a la LOMCE se incorporan otros elementos como textos literarios o historiográficos que mencionan o describen los elementos patrimoniales asociados al momento histórico descrito.

Estas fuentes diversas suelen ir asociadas a los hipertextos introducidos en los libros de texto. En este sentido es de destacar cómo el análisis realizado parece vincular cada vez más el elemento patrimonial a estos hipertextos. De hecho, entre el $52.2 \%$ y el $53.8 \%$ de los elementos patrimoniales incorporados en los libros de texto LOMCE aparecían vinculados a un hipertexto, que complementa el discurso más o menos homogéneo del texto principal. Su inclusión a modo de hipertexto permite romper el discurso lineal tradicional, lograr que el libro se convierta en un objeto más ágil, con diversidad de recursos asociados al mismo y que, además, se adapte a las nuevas formas de aproximación al texto escrito de los estudiantes como nativos digitales que son (Prensky, 2001). Este hecho, en principio, podría resultar positivo a la hora de concebir el patrimonio como algo más que un mero ilustrador de contenidos, pero los datos no parecen mostrarlo así, como se explica a continuación.

Ya estén vinculados a textos, hipertextos o acompañen a una actividad, las acciones demandadas sobre los elementos patrimoniales analizados han sido y son usualmente introducidas por verbos asociados a la identificación y descripción de los mismos, que demandan un bajo aprovechamiento cognitivo por parte del alumnado (con una presencia del $54.9 \%$ en los libros de texto LOE y un $46.7 \%$ en LOMCE). Pese al aumento de la presencia de actividades con una mayor demanda cognitiva en los libros de texto LOMCE, asociadas al hecho de que el alumnado tenga que analizar o investigar (y por lo tanto evaluar y crear nueva información), estas siguen siendo poco frecuentes, no superando el $9.4 \%$ de las actividades presentes en los libros de texto. Estos resultados son similares a los obtenidos por Sáiz (2014): en las actividades presentes en los manuales de Historia que hacían uso de fuentes primarias, un $58.7 \%$ de registros se correspondían con un nivel de aprovechamiento cognitivo bajo, un $39.6 \%$ con un nivel medio y un $1.6 \%$ con un nivel alto. También Gómez, Ortuño y Gandía (2013) concluían que en los libros de $2 .^{\circ}$ ESO el $47 \%$ de las actividades analizadas se limitaban a pedir la reproducción de información.

Por lo tanto, el perceptible aumento de fuentes y documentos históricos en los manuales escolares que ha tenido lugar durante las últimas dos décadas (Valls y López, 2010) no se ve todavía correspondido con un mayor aprovechamiento del potencial didáctico (y motivacional) de los recursos que estos contienen.

\section{Reflexiones finales}

Tras lo expuesto, se puede observar cómo a pesar de la integración del 
patrimonio arqueológico en los libros de texto, aún se está lejos de poder considerarlo como un recurso útil para la construcción de conocimiento que supere la mera lectura estética y que anime al alumnado a indagar sobre el pasado a través de sus vestigios. Como ya señalara López (2014) la falta de aprovechamiento del potencial del patrimonio en los libros de texto es notable.

Aún así, la cada vez mayor presencia del elemento patrimonial, su cada vez más usual inclusión a partir de múltiples formatos (entre ellos los objetos), su introducción a partir de fuentes diversas, como son las textuales, y el ligero aumento de actividades con una demanda cognitiva superior, delatan ciertos avances, lentos eso sí, pero constantes.

A pesar de que los datos aquí presentados y analizados ilustren y tengan validez para un marco geográfico y en un segmento temporal concretos, sirven para hacer más completa la imagen de conjunto a nivel estatal. Sería interesante aplicar este modelo de análisis a otras realidades geográficas e incluso editoriales, así como diversificar el estudio a otros elementos constituyentes de la enseñanza patrimonial (percepciones de los estudiantes, estrategias docentes o el papel de espacios fuera del aula). Pero sin duda alguna, esta investigación debería servir como base para saltar a una fase más propositiva, en la que se elaboren estrategias y proyectos cuya utilidad didáctica respecto al patrimonio material complete las deficiencias que los libros de texto parecen poseer y permitan integrar los recursos patrimoniales en el aula de una manera más activa, reflexiva y crítica.

\section{Referencias bibliográficas}

ADIEGO, P., BELLÓN, Á., FERNÁNDEZ, M. Á, LÓPEZ, R., RUBALCABA, R., VELILLA, J. y ZABALETA, F. J. (2015). Geografía e Historia, 1 ESO. Región de Murcia. Serie descubre. Proyecto Saber Hacer (Vol. 2). Madrid: Santillana.

ALBET, A., BENEJAM, P., GARCÍA, M. y GATELL, C. (2007). Ciencias sociales, geografía e historia. Educación secundaria. Primer curso. Demos. Región de Murcia. Vicens Vives.

ALBET, A., BOSCH, D., GARCÍA, C., GONZÁLEZ-MONFORT, N., GARCÍA, M. y GATELL, C. (2015). GH. Geografía e Historia. 1.2. Aula 3D (Vol. 2). Vicens Vives.

ANDERSON, L. W. y KRATHWOHL, D. R. (2001). A taxonomy for learning, teaching, and assessing: a revision of Bloom's taxonomy of educational objectives. New York: Longman.

ANDREU, J. (2002). Las técnicas de análisis de contenido: una revisión actualizada. Sevilla: Centro de Estudios Andaluces.

BARDAVIO, A., GATELL, C. y GONZÁLEZ, P. (2004). Is archaeology what matters? Creating a sense of local identity among teenagers in Catalonia. World Archaeology, 36(2), 261274.

BARDAVIO, A. y GONZÁLEZ, P. (2003). Objetos en el tiempo: las fuentes materiales en la enseñanza de las ciencias 
sociales. Barcelona: ICE Universitat de Barcelona : Horsori.

BEL, J. C. (2017). Imagen y libros de texto de Historia en Educación Primaria: estudio comparativo a partir de un análisis cualitativo. Revista de Educación, 377, 82-110. DOI: 10.4438/1988592X-RE-2017-377-354

BLOOM, B. S. (1956). Taxonomy of educational objectives: the classification of educational goals. Handbook 1, Cognitive domain. Reading, MA: Addison-Wesley Longman.

BURGUERA, J. (2006). Usos i abusos del llibre de text. Perspectiva Escolar, 302, 75-79.

CARRASCO, C., FIGUEIRA, M. D., GONZÁLEZ, G., GONZÁLEZ, J. L., MARCOS, A., SIERRA, G. y TORRES, F. (2007). Ciencias sociales. Geografía e Historia. $1^{o}$ secundaria. Proyecto ánfora. Madrid: Oxford University Press.

CORBISHLEY, M. (2011). Pinning down the past: archaeology, heritage, and education today. Woodbridge, UKRochester, NY: Boydell Press.

CUENCA, J. M. (2002). El patrimonio en la didáctica de las ciencias sociales: análisis de concepciones, dificultades y obstáculos para su integración en la enseñanza obligatoria. Universidad de Huelva, Huelva. Recuperado de http://rabida.uhu.es/dspace/handle/10272/2648 [10 de agosto de 2017]

CUENCA, J. M. y ESTEPA, J. (2003). El patrimonio en las Ciencias Sociales: Concepciones transmitidas por los libros de texto de E.S.O. En E. BALLESTEROS, C. FERNÁNDEZ, J. A. MOLINA y P. MORENO (Eds.), El patrimonio y la didáctica de las ciencias sociales (91-102). Cuenca: AUPDCS.

CUENCA, J. M., ESTEPA, J. y MARTÍN, M. M. (2017). Patrimonio, educación, identidad y ciudadanía. Profesorado y libros de texto en la enseñanza obligatoria. Revista de Educación, 375, 136-159. DOI: $10.4438 / 1988-592 X-$ RE-2016-375-338

CUENCA, J. M. y LÓPEZ, I. (2014). La enseñanza del patrimonio en los libros de texto de Ciencias Sociales, Geografía e Historia para ESO. Cultura y Educación, 26(1), 1-43. DOI: 10.1080/11356405.2014.908663

DURBIN, G., MORRIS, S. y WILKINSON, S. (1990). A teacher's guide to learning from objects. London: English Heritage.

EGEA, A. y ARIAS, L. (2013). IES Arqueológico. La arqueología como recurso para trabajar las competencias básicas en la Educación Secundaria. CLIO. History and History Teaching, 39. Recuperado de http://clio. rediris.es/n39/recursos/egeaarias.pdf [12 de julio de 2017]

EGEA, A., ARIAS, L. (2018). El desafío de enseñar a pensar históricamente a través de la arqueología. En A. EGEA, L. ARIAS y J. SANTACANA (Eds.), $Y$ la arqueología llegó al aula... La cultura material y el método arqueológico para la enseñanza de la historia y el patrimonio (327-341). Gijón: Trea. 
EGEA, A., ARIAS, L. y PERNAS, S. (2017). ¿Hay vida más allá de la arqueología? La educación como una oportunidad. Revista Temporis[Ação], 17(1), 20-42.

EGEA, A., PERNAS, S. y ARIAS, L. (2014). Re-construyendo la historia a partir del patrimonio arqueológico. En O. FONTAL, A. IBÁÑEZ y L. MARTÍN (Eds.), Reflexionar desde las experiencias. Una visión complementaria entre España, Francia y Brasil. Actas del II Congreso Internacional de Educación Patrimonial. Madrid: IPCE/OEPE.

ESTEPA, J., FERRERAS, M., LÓPEZ, I. y MORÓN, H. (2015). Análisis del patrimonio presente en los libros de texto: obstáculos, dificultades y propuestas. Revista de Educación, 355, 573-589.

FELIU, M. y HERNÀNDEZ, F. X. (2011). Enseñar y aprender historia: 12 ideas clave. Barcelona: Graó.

FERRERAS, M. y JIMÉNEZ, R. (2013). ¿Cómo se conceptualiza el patrimonio en los libros de texto de Educación Primaria? Revista de Educación, 361, 591-608. DOI: 10.4438/1988-592XRE-2013-361-234

GÁMEZ, V. (2016). La función educativa de la imagen en el área de Ciencias Sociales de Educación Primaria. Un estudio exploratorio en torno al currículo escolar, los libros d texto y la concepción del alumnado del Grado de Maestro de Educación Primaria. Universitat de Barcelona, Barcelona.
Recuperado de http://www.tdx.cat/ handle/10803/398013

GÓMEZ, C. J., CÓZAR, R. y MIRALLES, P. (2014). La enseñanza de la historia y el análisis de libros de texto. Construcción de identidades y desarrollo de competencias. Ensayos: Revista de La Facultad de Educación de Albacete, 29(1), 1-25.

GÓMEZ, C. J, ORTUÑO, J. y GANDÍA, E. (2013). La Edad Media en las clases de historia en ESO. Un análisis de las actividades de los libros de texto. En J. PRATS, I. BARCA y R. FACAL (coords.), História e Identidades culturales (445-457). Universidade do Minho.

GRENCE, T., CABALLERO, J. M., ESPINO, O., ETXEBARRIA, L., MARTÍN, G., PERALES, A., PRIETO, G. y RAMÍEZ, D. (2011). Geografía e Historia, 1 ESO. Región de Murcia (Vol. 2). Madrid: Santillana.

HENSON, D. (2004). Archaeology in Schools. En D. HENSON, P. G. STONE, y M. CORBISHLEY (Eds.), Education and the historic environment (23-32). Londres-New York: Routledge.

HENSON, D. (2009). The true end of archaeology? Primary History, 51, 7.

HERNÀNDEZ, F. X. (2003). El patrimonio como recurso en la enseñanza de las Ciencias Sociales. En E. BALLESTEROS, C. FERNÁNDEZ, J. A. MOLINA y P. MORENO (Eds.), El patrimonio y la didáctica de las ciencias sociales (455-466). Cuenca: AUPDCS. 
HERNÁNDEZ, A. M. ${ }^{\mathrm{a}}$ y GUILLÉN, R. (2017). La educación patrimonial en los manuales escolares de Educación Primaria: un recorrido desde LOGSE hasta LOMCE. Didáctica de las ciencias experimentales y sociales, 32(2), 25-49. DOI: 10.7203/DCES.32.9205

LÓPEZ, I. (2014). La educación patrimonial. Análisis de tratamiento didáctico del patrimonio en los libros de texto de CCSS en la Enseñanza Secundaria. Huelva: Universidad de Huelva.

LÓPEZ, I. y CUENCA, J. M. (2011). El patrimonio en los libros de texto de Ciencias Sociales de ESO: evaluación y nuevas perspectivas. En P. MIRALLES, S. MOLINA y A. SANTIESTEBAN (Eds.), La evaluación en el proceso de enseñanza y aprendizaje de las Ciencias Sociales (Vol. 2, 160168). Murcia: AUPDCS.

MARTÍNEZ, N. (2011). El uso de los manuales escolares de historia de España. Iber. Didáctica de Las Ciencias Sociales, Geografía e Historia, $70,48-58$.

MESEGUER, A. J., CABALLERO, E., ARIAS, L. y EGEA, A. (2018). ¿Hay un hueco para la arqueología en la realidad educativa? Tres pilares fundamentales para cambiar un modelo: leyes educativas, libros de texto y profesorado. En A. EGEA, L. ARIAS y J. SANTACANA (Eds.). Y la arqueología llegó al aula... La cultura material y el método arqueológico para la enseñanza de la historia y el patrimonio (25-44). Gijón: Trea.
NAVARRO,Á. y ALCOLEA, M.Á. (2015). Geografía e Historia. 1 ESO (Vol. 1). Madrid: Oxford University Press España.

PALLARÉS, M., CARRIÓN, A., y SÁNCHEZ, N. (2018). Trabajo por competencias en Educación Secundaria a través de una experiencia arqueológica. En A. EGEA, L. ARIAS y J. SANTACANA (Eds.). Y la arqueología llegó al aula... La cultura material y el método arqueológico para la enseñanza de la historia y el patrimonio (99-118). Gijón: Trea.

PRATS, J. (2012). Criterios para la elección del libro de texto de historia. Iber. Didáctica de las Ciencias Sociales, Geografía e Historia, 70, 7-13.

PRENSKY, M. (2001). Digital Natives, Digital Immigrants Part 1. On the Horizon, 9(5), 1-6. DOI: 10.1108/10748120110424816

RAJA, M. J. y MIRALLES, P. (2015). La enseñanza de la Geografía Física en los libros de texto de educación secundaria: de la Ley General de Educación a la Ley Orgánica de Educación. Didáctica Geográfica, 14, 109-128.

RAUSELL, H. (2017). ¿Cómo se enseña la Ilustración en $2 .^{\circ}$ de Bachillerato? Un análisis de los libros de texto, sus contenidos y la cuestión de género. Panta Rei. Revista Digital de Ciencia y Didáctica de la Historia, 109-122. DOI: $10.6018 /$ pantarei/2017/7

RÜSEN, J. (1997). El libro de texto ideal. Reflexiones en torno a los medios para guiar las clases de historia. Iber. 
Didáctica de Las Ciencias Sociales, Geografía e Historia, 12, 79-93.

SÁIZ, J. (2011). Actividades de libros de texto de Historia, competencias básicas y destrezas cognitivas, una difícil relación: análisis de manuales de $1^{\circ} \mathrm{y}$ $2^{\circ}$ de ESO. Didáctica de las Ciencias Experimentales y Sociales, 25, 37-64. SÁIZ, J. (2013). Alfabetización histórica y competencias básicas en libros de texto de historia y en aprendizaje de los estudiantes. Didáctica de las Ciencias Experimentales y Sociales, 27, 43-66. DOI: 10.7203/DCES.27.2648

SÁIZ, J. (2014). Fuentes históricas y libros de texto en secundaria: una oportunidad perdida para enseñar competencias de pensamiento histórico. Ensayos: Revista de La Facultad de Educación de Albacete, 29(1), 83-99.

SÁIZ, J., y COLOMER, J. C. (2014). ¿Se enseña pensamiento histórico en libros de texto de Educación Primaria? Análisis de actividades de historia para alumnos de 10-12 años de edad. Clio: History and History Teaching, 40. Recuperado de: http:// clio.rediris.es/n40/articulos/saizycolomer2014.pdf [17 de julio de 2017]

SÁNCHEZ, R., ARIAS, L. y EGEA, A. (2016). The Perduration of Master Narratives: The `Discovery', Conquest and Colonization of America in the Spanish Textbooks. The International Journal of Historical Teaching, Learning and Research, 13(2), 127-137.

SÁNCHEZ, R. y MARTÍNEZ, A. A. (2015). Patrimonio e identidad en la
Región de Murcia: una aproximación a través del currículo y los libros de texto de Ciencias Sociales de Secundaria. Clio. History and History Teaching, 41. Recuperado de: http://clio. rediris.es/n41/articulos/mono/MonSanchez2015.pdf [20 de julio de 2017]

SANTACANA, J. (1997). L'arqueologia com a eina didáctica: problemes i utilitat. Cota Zero, 15, 63-73.

SANTACANA, J. (2015). El patrimonio, la educación y el factor emocional. En Solé, G. (Ed.), Educação patrimonial: contributos para a construção de uma consciencia patrimonial (17-34). Braga: Centro de Investigação em Educação.

SANTACANA, J. y LLONCH, N. (2012). Manual de didáctica del objeto en el museo. Gijón: Trea.

SCHUG, M. C., WESTERN, R. D. y ENOCHS, L. G. (1997). Why do Social Studies Teachers Use Textbooks? The Answer May Lie in Economic Theory. Social Education, 62(1), 97-101.

SEIXAS, P. y MORTON, T. (2013). The Big Six Historical Thinking Concepts. Toronto: Nelson Education.

TRABAJO, M. y CUENCA, J. M. (2014). El patrimonio en la enseñanza de las ciencias sociales: una visión integrada del concepto de patrimonio; currículum, libro de texto, docente y estudiante. En O. FONTAL, A. IBÁÑEZ y L. MARTÍN (Eds.), Actas Del II Congreso Internacional de Educación Patrimonial. Madrid: MECD - OEPD.

VALLS, R. (1998). Los manuales escolares y los materiales curriculares de 
historia. Una propuesta de análisis y valoración crítica de los mismos desde criterios didáctico-historiográficos. Iber. Didáctica de Las Ciencias Sociales, Geografía e Historia, 17, 69-78.

VALLS, R. (1999). Sobre la elección y usos de las imágenes de los manuales escolares de historia: un ejemplo español (1900-1998). Clío y asociados. La historia enseñada, 4, 77- 100.

VALLS, R. (2001). Los estudios sobre manuales escolares de historia y sus nuevas perspectivas. Didáctica de las Ciencias Experimentales y Sociales, 15, 23-36.

VALLS, R. (2008). Los textos escolares de historia: una propuesta de análisis y valoración. En J. PRATS y M. ALBERT (Eds.), Ells llibres de text $i$ l'ensenyament de la Història (63-72). Barcelona: Universidad de Barcelona.

VALLS, R. y LÓPEZ, R. (2010). ¿Un nuevo paradigma para la enseñanza de la historia? Los problemas reales y las polémicas interesadas al respecto en España y en el contexto del mundo occidental. Enseñanza de las ciencias sociales, 10, 75-85.

WEARING, J., CASE, R., y EDWARDS,C. (2011). Teaching archaeological thinking: a professional resource to help teach six interrelated concepts central to students' ability to think critically about archaeology. Vancouver: Critical Thinking Consortium.

\section{Documentos legislativos}

Convenio Europeo sobre la Protección del Patrimonio Arqueológico realizado en La Valetta el 16 de enero de 1992 y revisado y ratificado por el Gobierno de España el 20 de julio de 2011. Boletín Oficial del Estado (BOE), 173, Sección I, 12500-125001.

Ley Orgánica 1/1990, de 3 de octubre, de Ordenación General del Sistema Educativo. Boletín Oficial del Estado (BOE), 238, de 4 de octubre de 1990, 28927-28942.

Ley Orgánica 2/2006, de 3 de mayo, de Educación. Boletín Oficial del Estado (BOE), 106, de 4 de septiembre de 2006, 17158-17207.

Ley Orgánica 8/2013, de 9 de diciembre, para la mejora de la calidad educativa. Boletín Oficial del Estado (BOE), 295, de 10 de diciembre de 2013, 97858-97921.

Ley 16/1985, de 25 de junio, del Patrimonio Histórico Español. Boletín Oficial del Estado (BOE), Madrid, 29 de junio de 1985.

UNESCO (1990). Charter for the Protection and Management of Archaeological Heritage. Recuperado de: https:// www.icomos.org/images/DOCU MENTS/Charters/arch_e.pdf [20 de junio de 2017] 